\title{
A Modified Nonsmooth Levenberg-Marquardt Algorithm for the General Mixed Complementarity Problem
}

\author{
Linsen Song $\mathbb{D}^{1,2}$ and Yan Gao ${ }^{3}$ \\ ${ }^{1}$ College of Mathematics and Information Science, Henan Normal University, Xinxiang 453003, China \\ ${ }^{2}$ School of Mathematical Sciences, Henan Institute of Science and Technology, Xinxiang 453003, China \\ ${ }^{3}$ School of Management, University of Shanghai for Science and Technology, Shanghai 200093, China
}

Correspondence should be addressed to Linsen Song; slinsen@163.com

Received 7 July 2021; Revised 27 October 2021; Accepted 27 October 2021; Published 16 November 2021

Academic Editor: Qiuye Sun

Copyright ( 92021 Linsen Song and Yan Gao. This is an open access article distributed under the Creative Commons Attribution License, which permits unrestricted use, distribution, and reproduction in any medium, provided the original work is properly cited.

\begin{abstract}
As is well known, the mixed complementarity problem is equivalent to a nonsmooth equation by using a median function. By investigating the generalized Jacobi of a composite vector-valued maximum function, a nonsmooth Levenberg-Marquardt algorithm is proposed in this paper. In the present algorithm, we adopt a new LM parameter form and discuss the local convergence rate under the local error bound condition, which is weaker than nonsingularity. Finally, the numerical experiments and the application for the real-time pricing in smart grid illustrate the effectiveness of the algorithm.
\end{abstract}

\section{Introduction}

1.1. Background and Motivation. Mixed complementarity problem, MCP for short, is one of the important types of finite dimensional variational inequality, such that to find a vector $x \in \mathbb{R}^{n}$ as follows:

$$
F(x)^{T}(y-x) \geq 0, \quad \forall y \in X,
$$

where $F(x)=\left(F_{1}(x), \ldots, F_{n}(x)\right)^{T}$ is assumed to be continuously differentiable, $X=\prod_{i=1}^{n}\left[l_{i}, u_{i}\right],-\infty<l_{i}<u_{i}<$ $+\infty, i=1, \ldots, n$. While $l_{i}=0, u_{i}=\infty$, the MCP is the nonlinear complementarity problem (NCP). The MCP is extensively used in the field of science and engineering, where the practical problem is transformed into the MCP firstly and then is investigated for its solvability and the stability of the solution [1-7], such as the real-time pricing in smart grid, which will be the application of our present algorithm.

Recently, the real-time pricing (RTP) principle, which is determined before the deal happening through the intercommunication between the supply and demand of electricity and can guide and motivate the users in off-peak electricity by adjusting the real-time price signal so as to reduce the peak load, achieves the purpose of peak cutting and has been widely recognized as one of the promising solutions to adjust the power balance between the supply and demand in smart grid system. A decade ago, Samadi et al. proposed a RTP model called social welfare maximization model (SWMM) to maximize the aggregated welfare for the users as well as minimizing the cost of the providers [8-10], based on which some different optimization algorithms have been proposed [11-13]. These algorithms are proved feasible and effective by the numerical results. However, these are most smoothing methods, where the gaps exist between the approximate smoothing system and the original nonsmooth system so that the solution may be not as accurate as nonsmooth algorithms. Considering that, may be transforming the practical model to the mixed complementarity problem and investigating its nonsmooth algorithm are meaningful and valuable.

1.2. Related Work and Challenge. In the last years, many results on the theories and algorithms for the MCP have been developed [14-16]. One of the most popular 
approaches for the MCP is to reformulate it as an equivalent equation such that

$$
H(x)=0,
$$

where

$$
H_{i}(x)=x_{i}-\operatorname{mid}\left(l_{i}, u_{i}, x_{i}-F_{i}(x)\right),
$$

and $\operatorname{mid}(a, b, c)$ represents the median of the three scalar $a, b, c$. Obviously, the system of equation (2) is nonsmooth. Since the subdifferential of vector-valued median function is not easy to obtain, smoothing methods have received much attentions based on some smoothing functions with good property for MCP. Gabriel and Moré proposed a class of smoothing functions for the median function [14]. By far, the three most often used functions are the networks function, the Chen-Harker-Kanzow-Smale (CHKS) smoothing function and the uniform smoothing function. Zhou and Peng proposed new class smoothing functions, called the locally Chen-Harker-Kanzow-Smale (LCHKS) smoothing functions for the MCP [15]. Chen et al. studied the Jacobian smoothing Newton method and established superlinear convergence of a smoothing Newton method [16]. Qi et al. proposed a modified smoothing Newton method based on a $2 n$-dimensional semismooth equation reformulation [17]. These algorithms are proved feasible and effective by the numerical results. However, the nonsmooth algorithms for (2), which may be more accurate and more suitable for the practical problem, are few discussed by our knowledge. Considering that, if the algorithm for the subdifferential of vector-valued median function is obtained, the nonsmooth algorithms for MCP are more effective. Hence, we focus on a nonsmooth Levenberg-Marquardt method, namely, a modified Newton method, with a new parameter form by investigating a maximum-valued composition function's subdifferential in this paper. Also, the numerical experiments and the application for the real-time pricing in smart grid were given to illustrate the effectiveness of the algorithm.

The outline of the paper is as follows. In Section 2, we recall some preliminaries in nonsmooth analysis and propositions necessary. In Section 3, a nonsmooth Levenberg-Marquardt method is present based on the computation of a maximum-valued composition function's subdifferential, also its local convergence rate under the local error bound, which is weaker than nonsingularity is shown. In Section 4, the numerical experiments illustrate the effectiveness of the algorithm. In Section 5, we apply the present algorithm to the real-time pricing in smart grid, which also illustrates the present algorithm's effectiveness when compared with the traditional fixed price method.

\section{Preliminaries}

In what follows, we recall some basic concepts and propositions necessary in this paper.

In nonsmooth analysis, there are three common forms of differential, such as B-differential denoted as $\partial_{B}(\cdot)$, Clarke generalized Jacobian denoted as $\partial_{\mathrm{Cl}}(\cdot)$, and C-subdifferential denoted as $\partial_{\mathrm{Cl}}(\cdot)$, where $\partial_{B} H(x) \subseteq \partial_{\mathrm{Cl}} H(x) \subseteq \partial_{\mathrm{C}} H(x)$.
Let $H: \mathbb{R}^{n} \longrightarrow \mathbb{R}^{m}$ be locally Lipschitzian; $\Omega_{H}$ denotes a set, where $H$ is not differential; B-differential of $H$ at $x \in \mathbb{R}^{n}$ is defined by

$$
\partial_{B} H(x)=\left\{\lim \mathrm{JH}\left(x_{i}\right): x_{i} \longrightarrow x, x_{i} \notin \Omega_{H}\right\} .
$$

Clarke generalized Jacobian of $H$ at $x \in \mathbb{R}^{n}$ is defined as the convex hull of $\partial_{B} H(x)$ such that

$$
\partial_{\mathrm{Cl}} H(x)=\operatorname{conv} \partial_{B} H\left(x_{k}\right) .
$$

C-subdifferential of $H$ at $x \in \mathbb{R}^{n}$ is defined by

$$
\partial_{\mathrm{C}} H(x)=\partial_{\mathrm{Cl}} H_{1}(x) \times \cdots \times \partial_{\mathrm{Cl}} H_{m}(x),
$$

where $H_{i}(x)$ is the $i$ th component of the function $H(x)$.

Subdifferentially regular function is a subclass of Lipschitzian functions. For function $H: \mathbb{R}^{n} \longrightarrow \mathbb{R}$, if it is locally Lipschitz continuous at $x$ and its classical directional derivative $H^{\prime}(x ; d)$ exists and there is

$$
H^{\prime}(x ; d)=H^{o}(x ; d)
$$

for all $d \in \mathbb{R}^{n}$, we say it is subdifferentially regular at $x$. The subdifferentially regular function is broad and includes many types, such as smooth functions and convex functions, if $f=\sum_{i=1}^{m}(x) \lambda_{i} f_{i}$, where $\lambda_{i}>0$ and $f_{i}$ is subdifferentially regular at $x$ for each $i=1, \ldots, m$.

Subdifferentially regular functions have some special properties such that

(i) If $f_{i}: \mathbb{R}^{n} \longrightarrow \mathbb{R}^{m}$ is subdifferentially regular at $x$, there is $\partial f(x)=\lambda_{i} \partial f_{i}(x)$.

(ii) Let $f: \mathbb{R}^{n} \longrightarrow \mathbb{R}$ be such that $f=g^{\circ} h$, where $h: \mathbb{R}^{n} \longrightarrow \mathbb{R}^{m}, g: \mathbb{R}^{m} \longrightarrow \mathbb{R}$. If the function $g$ is subdifferentially regular at $h(x)$, each $h_{i}$ is subdifferentially regular at $x$, there is $\partial f(x)=$ conv $\left\{\partial h(x)^{T} \partial g(h(x))\right\}$.

Subdifferentially regular functions are always semismooth, which includes many types of functions, such as smooth functions, maximum functions, and so on. There are some properties for semismooth functions such that

(i) $V d-H^{\prime}(x ; d)=o(d), \forall V \in \partial_{\mathrm{Cl}} H(x+d)$.

(ii) $H(y)-H(x)-V^{T}(y-x)=o(\|y-x\|), \forall V \in \partial_{\mathrm{Cl}}$ $H(x)$.

Obviously, since $\partial_{B} H(x) \subseteq \partial_{\mathrm{Cl}} H(x)$, the properties also exist when $\forall V \in \partial_{B} H(x)$.

\section{The Algorithm and Its Local Convergence Rate}

In this section, we give a modified nonsmooth Levenberg-Marquardt method based on the calculation of an element in Clarke Jacobi of a composite vector-valued maximum function firstly and then the local convergence rate under the local error bound condition was investigated.

The Levenberg-Marquardt method (LM) algorithm is a classical and popular approach for solving nonlinear equations, which is also called an inexacted Newton method. It is used to solve the nonsmooth equations as follows: 


$$
x_{k+1}=x_{k}-\left(\xi_{k}^{T} \xi_{k}+\lambda_{k} I\right)^{-1} \xi_{k}^{T} H\left(x_{k}\right) \text {, }
$$

where $\xi_{k}:=\xi\left(x_{k}\right) \in \partial_{B} H\left(x_{k}\right), \lambda_{k}>0$, is called LM parameter. In general, it is not easy to calculate one element in $\partial_{B} H\left(x_{k}\right)$, which is necessary to ensure the Newton-types algorithms operation. In the LM methods, the LM parameter is important, different forms of the parameters may cause different convergent rate. In [18], Fan showed the LM parameter $\lambda_{k}=\mu_{k}\left\|H_{k}\right\|^{\delta}$ with $\delta \in(0,2]$, where $\mu_{k}$ is updated by trust region techniques to make the algorithm still have good results when the sequence is far away from the solution. However, Amini and Rostami [19] thought the LM method with $\lambda_{k}=\mu_{k}\left\|H_{k}\right\|^{\delta}$ and $\delta=1$ may reverse outcome on the result of initial steps which are far from the solution set. They thought $\left\|H_{k}\right\|$ may be so large that the LM step is small, which can reduce the efficiency of the algorithm. They proposed a new LM parameter as follows:

$$
\lambda_{k}=\frac{\mu_{k}\left\|H_{k}\right\|}{1+\left\|H_{k}\right\|} \text {. }
$$

Inspired by this and considering the above algorithms are all for the equations, where $H(x)$ is continuously differentiable, we adopted the LM parameter in the nonsmooth LM algorithm such that

$$
\lambda_{k}=\frac{\mu_{k}\left\|H_{k}\right\|^{\delta}}{1+\left\|H_{k}\right\|^{\delta}},
$$

where $\delta \in(0,2]$. We next give the modified nonsmooth LM algorithm.

Remark 1. It is noticeable that one element of the function $H^{\prime}$ s B-differential, namely, the Clarke Jacobi is necessary in Algorithm 1. Hence, a subalgorithm is the basis to run Algorithm 1.

In what follows, we investigate the calculation of one element of the $H^{\prime}$ s Clarke Jacobi.

Since $H_{i}(x)=\operatorname{mid}\left(l_{i}, u_{i}, x_{i}-F_{i}(x)\right)$ in (2), and there is

$$
\begin{aligned}
H_{i}(x)= & F_{i}(x)-l_{i}-u_{i}+\min \left\{l_{i}, u_{i}, x_{i}-F_{i}(x)\right\} \\
& +\max \left\{l_{i}, u_{i}, x_{i}-F_{i}(x)\right\},
\end{aligned}
$$

namely,

$$
\begin{aligned}
H_{i}(x)= & F_{i}(x)-l_{i}-u_{i}-\max \left\{-l_{i}, F_{i}(x)-x_{i}\right\} \\
& +\max \left\{u_{i}, x_{i}-F_{i}(x)\right\} .
\end{aligned}
$$

The function $H$ is actually a composite vector-valued maximum function. We focus on the B-differential of a composite vector-valued maximum function for general form such that

$$
G(x)=\left(\begin{array}{c}
g_{1}\left(\max _{j \in J_{1}^{(1)}} f_{1 j}^{(1)}(x), \ldots, \max _{j \in J_{m}^{(1)}} f_{m j}^{(1)}(x)\right) \\
\vdots \\
g_{n}\left(\max _{j \in J_{1}^{(n)}} f_{1 j}^{(n)}(x), \ldots, \max _{j \in J_{m}^{(n)}} f_{m j}^{(n)}(x)\right)
\end{array}\right),
$$

where $g_{s}: \mathbb{R}^{m} \longrightarrow \mathbb{R}$ for $s=1, \ldots, n, f_{k j}^{(s)}: \mathbb{R}^{n} \longrightarrow \mathbb{R}$ for $j \in J_{k}^{(s)}, k=1, \ldots, m$, is continuously differentiable, and $J_{k}^{(s)}$ for $k=1, \ldots, m$ are finite index sets. Let $h_{i}=\left(\max _{j \in J_{i}^{(i)}}\right.$ $\left.f_{i j}^{(i)}(x), \ldots, \max _{j \in J_{m}^{(i)}} f_{m j}^{(i)}(x)\right)$; the function $G(x)$ can be denoted as

$$
G(x)=\left(g_{1}\left(h_{1}(x)\right), \ldots, g_{n}\left(h_{n}(x)\right)^{T}\right) .
$$

Denote

$$
\begin{gathered}
S_{t}^{(i)}(x)=\left\{k: f_{t k}^{(i)}(x)=\max _{s \in J_{t}^{(i)}} f_{t s}^{(i)}(x)\right\}, \\
i=1, \ldots, n, t=1, \ldots, m,
\end{gathered}
$$

and $\bar{S}_{t}^{(i)}(x)$ as a subset of $S_{t}^{(i)}(x)$ satisfying some properties such that

(i) For any $k \in S_{t}^{(i)}(x)$, there always exists $s \in \bar{S}_{t}^{(i)}(x)$ such that

$$
\nabla f_{t s}^{(i)}(x)=\nabla f_{t k}^{(i)}(x)
$$

(ii) For any $k, t \in \bar{S}_{t}^{(i)}(x), k \neq t$, there is

$$
\nabla f_{t s}^{(i)}(x) \neq \nabla f_{t s}^{(i)}(x)
$$

Lemma 1. Suppose $\bar{S}_{t}^{(i)}(x)$ is a singleton or there exists a nonnegative constant $a_{q_{t_{i}}}$ for $k_{i}$ such that

$$
\begin{aligned}
\nabla f_{t k_{i}}^{(i)}(x)-\nabla f_{t s}^{(i)}(x)= & \left(0, \ldots, a_{q_{t_{i}}}, \ldots, a_{n}\right)^{T}, \\
& \forall s \in \bar{S}_{t}^{(i)}(x), t=1, \ldots, m,
\end{aligned}
$$

then $\left(V_{1}, \ldots, V_{n}\right)^{T} \in \partial_{B} h_{i}(x)$, where $V_{i}=\left(f_{1 k_{1}}^{(i)}(x), \ldots\right.$, $\left.f_{m k_{m}}^{(i)}(x)\right), k_{j} \in \bar{S}_{j}^{(i)}(x), j=1, \ldots, m$.

Proof. The statement is obvious by the virtue of Theorem 1 in [20]; we omit the proof.

Theorem 1. Let $\left(V_{1}, \ldots, V_{n}\right)^{T} \in \partial_{B} h_{i}(x), g_{i}: \mathbb{R}^{m} \longrightarrow \mathbb{R}$ for $i=1, \ldots, n$ be continuously differentiable, then $\left(\nabla g_{1}, \ldots\right.$, $\left.\left.\nabla g_{n}\right)^{T} \in \partial_{B} G_{i}(x)\right)$, where $\nabla g_{i}=\left(V_{1}, \ldots, V_{n}\right)^{T \nabla} g_{i}\left(h_{i}(x)\right)$.

Proof. By the properties of the subdifferentially regular function and Lemma 1, we have

$$
\partial h_{i}(x)^{T} \partial g_{i}\left(h_{i}(x)\right) \in \partial_{B} G_{i}(x) \subset \nabla G_{1},
$$

we imply the statement.

We next study the local convergence properties of the present algorithm. Give some necessary assumptions firstly.

\section{Assumption 1}

(i) The solution set $X$ of MCP is nonempty, and the sequence $\left\{x_{k}\right\}$ converges to $X$, where some neighbourhoods of $x^{*} \in X$ lie in. 
Step 0 . Give an initial point $x_{0} \in \mathbb{R}^{n}$ and parameters $\varepsilon>0,0<m<1,0<M<\infty, 0<$ $p_{1}<p_{2}<1, \eta_{1} \in(0,1 / 2), \eta_{2} \in\left(\eta_{1}, 1\right], \eta_{3} \in[0,1 / 4), \mu_{0}>0$.

Step 1. Compute $H_{k}, V_{k}$. If $\left\|V_{k}^{T} H_{k}\right\| \leq \varepsilon$, stop. Otherwise, go to step 2.

Step 2. Set

$\lambda_{k}=\mu_{k}\left\|H_{k}\right\|^{\delta} / 1+\left\|H_{k}\right\|^{\delta}$,

where $\delta \in(0,2]$.

Step 3. Solve the following nonlinear equations to obtain $d_{k}$,

$\left(V_{k}^{T} V_{k}+\lambda_{k} I\right) d_{k}=-V_{k}^{T} H_{k}$,

where $V_{k} \in \partial_{B} H_{k}$.

Step 4. Compute $r_{k}=\operatorname{Ared}_{k} / \operatorname{Pred}_{k}$, where

Ared $_{k}:=\varphi\left(x_{k}+d_{k}\right)-\varphi\left(x_{k}\right)$

Pred $_{k}:=H_{k}^{T} V_{k} d_{k}+1 / 2 d_{k}^{T} V_{k}^{T} V_{k} d_{k}$

Step 5. If $r_{k}>\eta_{3}$, set $x_{k+1}=x_{k}+d_{k}$, else $x_{k+1}=x_{k}$.

Step 6. Update the parameter $\mu_{k}$ as follows

$\mu_{k+1}= \begin{cases}\min \left\{4 \mu_{k}, M\right\} & \text { if } r_{k}<p_{1} \\ \mu_{k} & \text { if } r_{k} \in\left[p_{1}, p_{2}\right] \\ \max \left\{0.25 \mu_{k}, m\right\} & \text { if } r_{k}>p_{2}\end{cases}$

Then, go to step 1 .

Algorithm 1: Algorithm NLM.

(ii) $\|H\|$ provides a local error bound on some neighborhood of $\bar{x} \in X$. Namely, there exists constants $c_{1}>0$ such that

$$
c_{1} \cdot \operatorname{dist}(x, X) \leq\|H(x)\|, \forall x \in N(\bar{x}, r),
$$

where $\operatorname{dist}(x, X)=\inf _{y \in X}\|y-\bar{x}\|$ and $N(\bar{x}, r)$ is a neighborhood of $\bar{x}$.

(iii) There exists a positive constant $L_{1}$ such that

$$
\|H(y)-H(x)\| \leq L_{1}\|y-x\|, \forall x, y \in N(\bar{x}, r) .
$$

Lemma 2. Let $\operatorname{dist}\left(x_{k}, X\right)=\left\|x_{k}-\overline{x_{k}}\right\|$, where $\overline{x_{k}} \in X$, under the conditions of Assumption 1, there is

$$
\left\|d_{k}\right\| \leq O\left(\left\|x_{k}-\overline{x_{k}}\right\|^{\alpha}\right),
$$

where $\alpha=\min \{1+p-\delta / 2,1\}$.

Proof. By virtue of the prosperities of the semismooth function $H(x)$, there is

$$
\left\|H(y)-H(x)-V^{T}(y-x)\right\| \leq L\|y-x\|^{1+p},
$$

where $L$ and $p$ are positive constants, respectively. Especially when $H$ is strongly semismooth, we have $p \geq 1$. Denote $q(d)=\left\|H_{k}+V_{k} d\right\|^{2}+\lambda_{k}\|d\|^{2}$. Similar to the proof of Lemma 4.1 in [20], it is obvious that

$$
\begin{aligned}
\left\|d_{k}\right\|^{2} & \leq \frac{1}{\lambda_{k}} q\left(d_{k}\right) \\
& \leq \frac{L^{2}\left\|\overline{x_{k}}-x_{k}\right\|^{2(1+p)}}{\lambda_{k}}+\left\|\overline{x_{k}}-x_{k}\right\|^{2} \\
& =\frac{L^{2}\left(1+\|H(x)\|^{\delta}\right)\left\|\overline{x_{k}}-x_{k}\right\|^{2(1+p)}}{\|H(x)\|^{\delta}}+\left\|\overline{x_{k}}-x_{k}\right\|^{2}
\end{aligned}
$$

$$
=\frac{L^{2}\left(1+L_{1}\left\|\overline{x_{k}}-x_{k}\right\|^{\delta}\right)\left\|\overline{x_{k}}-x_{k}\right\|^{2(1+p)}}{L_{1}\left\|\overline{x_{k}}-x_{k}\right\|^{\delta}}+\left\|\overline{x_{k}}-x_{k}\right\|^{2}
$$

which means that $\left\|d_{k}\right\| \leq O\left(\left\|x_{k}-\overline{x_{k}}\right\|^{\alpha}\right)$, where $\alpha=\min \{1+$ $p-\delta / 2,1\}$.

Theorem 2. Suppose the initial point $x_{0}$ is chosen sufficiently close to the solution set $X$, and $\left\{x_{k}\right\}$ is generated by Algorithm 1, then $\left\{x_{k}\right\}$ converges to some solution $x^{*}$ of the nonsmooth equation (2) with the convergence rate $\gamma$ under Assumption 1, where $\alpha \beta \leq \gamma \leq \beta$, and $\alpha=\min \{1+p-\delta /$ $2,1\}, \beta=\min \{1+p, 1+\delta / 2\}$.

Proof. The proof is similar to the proof of Theorem 4.1 in [20]. Combining (21) with (22) and (23), it is obvious that

$$
\begin{aligned}
\left\|H_{k}+V_{k} d_{k}\right\|^{2} \leq & q\left(d_{k}\right) \\
\leq & \left\|H_{k}+V_{k}\left(\overline{x_{k}}-x_{k}\right)\right\|^{2}+\lambda_{k}\left\|\overline{x_{k}}-x_{k}\right\|^{2} \\
= & \left\|H\left(x_{k}\right)-H\left(\overline{x_{k}}\right)-V_{k}\left(x_{k}-\overline{x_{k}}\right)\right\|^{2} \\
& +\frac{\mu_{k}\left\|H_{k}\right\|^{\delta}}{1+\left\|H_{k}\right\|^{\delta}}\left\|x_{k}-\overline{x_{k}}\right\|^{2} \\
= & \left\|H\left(x_{k}\right)-H\left(\overline{x_{k}}\right)-V_{k}\left(x_{k}-\overline{x_{k}}\right)\right\|^{2} \\
& +\mu_{k}\left\|H_{k}\right\|^{\delta}\left\|x_{k}-\overline{x_{k}}\right\|^{2} \\
\leq & L^{2}\left\|x_{k}-\overline{x_{k}}\right\|^{2(1+p)}+\mathrm{ML}_{1}^{\delta}\left\|x_{k}-\overline{x_{k}}\right\|^{2+\delta},
\end{aligned}
$$


which implies

$$
\left.\left\|H_{k}+V_{k} d_{k}\right\| \leq O\left(\| x_{k}-\overline{x_{k}}\right)^{\beta}\right),
$$

where $\beta=\min \{\{1+p, 1+\delta / 2\}$. Furthermore, since

$$
\left\|H\left(x_{k}+d_{k}\right)\right\| \leq\left\|H\left(x_{k}\right)+V_{k} d_{k}\right\|+L_{1}\left\|d_{k}\right\|^{1+p},
$$

holds by virtue of the property of the semismooth function $H$, we have

$$
\left\|H\left(x_{k}+d_{k}\right)\right\| \leq O\left(\left\|x_{k}-\overline{x_{k}}\right\|^{\beta}\right),
$$

that is to say, there exists a positive constant $c_{2}$ such that

$$
\left\|H\left(x_{k}+d_{k}\right)\right\| \leq c_{2} \operatorname{dist}\left(x_{k}, X\right)^{\beta} .
$$

Hence, combining (20) with (26), we have

$$
\operatorname{dist}\left(x_{k}+d_{k}, X\right) \leq \frac{1}{c_{1}}\left\|H\left(x_{k}+d_{k}\right)\right\| \leq \frac{c_{2}}{c_{1}} \operatorname{dist}\left(x_{k}, X\right)^{\beta} \text {. }
$$
that

Thus, combining (30) with the following inequality such

$$
\operatorname{dist}\left(x_{k}, X\right) \leq \operatorname{dist}\left(x_{k}+d_{k}, X\right)+\left\|d_{k}\right\|,
$$

which implies

$$
\operatorname{dist}\left(x_{k}, X\right) \leq 2\left\|d_{k}\right\|,
$$

for $k$ large enough and $\delta \in(0,2]$, we have

$$
\operatorname{dist}\left(x_{k}, X\right) \leq O\left(\left\|d_{k}\right\|^{\alpha}\right) \text {. }
$$

Furthermore, from (22), (30), and (32), there is

$$
\left\|d_{k+1}\right\|=O\left(\left\|d_{k}\right\|^{\gamma}\right)
$$

where $\alpha \beta \leq \gamma \leq \beta$.

\section{Numerical Experiments}

In this section, we compare the present algorithm (NLM) with one-step smoothing method (OSN) with global convergence proposed by Tang et al. in [21] at different initial points. Here, we set $\varepsilon=1.0 e-6, \mu_{0}=1, m=1.0 e-6, M=$ $1.0 e+8, \eta_{1}=0.001, \eta_{2}=1, \eta_{3}=0$ in NLM. The numerical results are listed in Tables 1-3, respectively. “-” denotes that the algorithm fails the solution.

Problem 1. Let

$$
F(x)=\left(\begin{array}{c}
x_{1}^{3}-8 \\
x_{2}-x_{3}+x_{2}^{3}+3 \\
x_{2}+x_{3}+2 x_{3}^{3}-3 \\
x_{4}+2 x_{4}^{3}
\end{array}\right)
$$

(1) Set $[l, u]=[0,5]^{4}$; the MCP has the solution $x^{*}=$ $(2,0,1,0)^{T}, F\left(x^{*}\right)=(0,2,0,0)^{T} ;(2)$ set $[l, u]=[-1,1]^{4}$; the
MCP has the solution $x^{*}=(1,-1,1,0)^{T}, F(x)=(-7,0$, $-1,0)^{T}$.

Problem 2. Let $F(x)=M x+q$, where

$$
M=\left(\begin{array}{cccc}
4 & 2 & 2 & 1 \\
2 & 4 & 0 & 1 \\
2 & 0 & 2 & 2 \\
-1 & -1 & -2 & 0
\end{array}\right), q=\left(\begin{array}{c}
-8 \\
-6 \\
-4 \\
3
\end{array}\right) .
$$

Set $[l, u]=[-1,1]^{4}$; the MCP has the solution $x^{*}=$ $(1,8 / 9,5 / 9,4 / 9)^{T}, F\left(x^{*}\right)=(-2 / 3,0,0,0)^{T} ;(2)$ set $[l, u]=$ $[-5,5]^{4}$; the MCP has the solution $x^{*}=(4 / 3,7 / 9,4 / 9$, $2 / 9)^{T}, F(x)=(0,0,0,0)^{T}$.

Problem 3 (Kojima-Shindo problem). Let $[l, u]=\left[0,10^{5}\right]^{4}$,

$$
F(x)=\left(\begin{array}{c}
3 x_{1}^{2}+2 x_{1} x_{2}+2 x_{2}^{2}+x_{3}+3 x_{4}-6 \\
2 x_{1}^{2}+x_{1}+x_{2}^{2}+10 x_{3}+2 x_{4}-2 \\
3 x_{1}^{2}+x_{1} x_{2}+2 x_{2}^{2}+2 x_{3}+9 x_{4}-9 \\
x_{1}^{2}+2 x_{2}^{2}+2 x_{3}+3 x_{4}-3
\end{array}\right) \text {. }
$$

There exists solutions $x^{*}=(\sqrt{6} / 2,0,0,1 / 2)^{T}, x^{* *}=$ $(1,0,3,0)^{T}$ in this problem.

\section{Application for Real-Time Pricing in Smart Grid}

Normally, suppose an electric power distribution system that consists of a single energy provider and $N$ load subscribers or users. An energy provider and all users are connected with each other through an information communication infrastructure. Divide a whole cycle into $K$ periods. Let $x_{i}(k)$ be the power consumption demand at time slot $k$ of the customer $i$.

The social welfare maximization model proposed by Samadi et al. [8] is as follows:

$$
\begin{array}{cl}
\max & \sum_{k=1}^{K}\left(\sum_{i=1}^{N} U\left(x_{i}(k), \omega_{i}(k)-C_{k}\left(L_{k}\right)\right),\right. \\
\text { s.t. } & \sum_{i \in N} x_{i}(k) \leq L_{k}, \quad k=1,2, \ldots, K, \\
& x_{i}(k) \geq 0, \quad k=1,2, \ldots, K,
\end{array}
$$

where $L_{k}$ means the production capacity of the energy provider at the time slot $k, C_{k}$ is the cost function of the energy provider, and the utility function $U\left(x_{i}(k), \omega_{i}(k)\right)$ is such that

$$
U_{i}\left(x_{i}(k), \omega_{i}(k)\right)= \begin{cases}\omega_{i}(k) x_{i}(k)-\frac{\alpha_{i} x_{i}(k)^{2}}{2}, & \text { if } 0 \leq x_{i}(k)<\frac{w_{i}(k)}{\alpha_{i}}, \\ \frac{w_{i}(k)^{2}}{2 \alpha_{i}}, & \text { if } x_{i}(k) \geq \frac{w_{i}(k)}{\alpha_{i}} .\end{cases}
$$


Table 1: Numerical results of Problem 1.

\begin{tabular}{|c|c|c|c|c|c|}
\hline \multirow{2}{*}[l,u]{} & \multirow{2}{*}{ Initial point } & \multicolumn{2}{|c|}{ OSN } & \multicolumn{2}{|c|}{ NLM } \\
\hline & & Iter & $\|H(x)\|$ & Iter & $\|H(x)\|$ \\
\hline \multirow{3}{*}[0,5]{} & $(1,3,2,4)^{T}$ & 17 & $2.6893 e-08$ & 10 & $1.9829 e-05$ \\
\hline & $(4,3,1,4)^{T}$ & 18 & $1.9489 e-07$ & 9 & $4.2584 e-12$ \\
\hline & $(5,5,5,5)^{T}$ & 24 & $1.9168 e-07$ & 14 & $1.4399 e-06$ \\
\hline \multirow{3}{*}[-1,1]{} & $(1,1,1,1)^{T}$ & 8 & $3.6500 e-04$ & 8 & $9.6985 e-06$ \\
\hline & $(1,-1,1,1)^{T}$ & 8 & $3.6500 e-04$ & 7 & $8.3019 e-07$ \\
\hline & $(1 / 2,-1 / 2,1,1)^{T}$ & 8 & $2.1316 e-04$ & 6 & $8.0830 e-06$ \\
\hline
\end{tabular}

TABLe 2: Numerical results of Problem 2.

\begin{tabular}{|c|c|c|c|c|c|}
\hline \multirow{2}{*}[1,u]{} & \multirow{2}{*}{ Initial point } & \multicolumn{2}{|c|}{ OSN } & \multicolumn{2}{|c|}{ NLM } \\
\hline & & Iter & $\|H(x)\|$ & Iter & $\|H(x)\|$ \\
\hline \multirow{3}{*}[-1,1]{} & $(1,1,1,0)^{T}$ & 8 & $1.7666 e-04$ & 5 & $2.9456 e-05$ \\
\hline & $(1,3,2,4)^{T}$ & 6 & $4.5967 e-07$ & 7 & $9.8310 e-05$ \\
\hline & $(4,3,1,4)^{T}$ & 9 & $3.2626 e-08$ & 12 & $1.0820 e-09$ \\
\hline \multirow{3}{*}[-5,5]{} & $(-1,-1,-1,-1)^{T}$ & 6 & $1.3938 e-08$ & 7 & $5.7825 e-05$ \\
\hline & $(2,4,3,5)^{T}$ & 6 & $1.3938 e-08$ & 8 & $5.2440 e-07$ \\
\hline & $(5,-5,-5,5)^{T}$ & 8 & $1.0340 e-08$ & 10 & $6.2649 e-07$ \\
\hline
\end{tabular}

Table 3: Numerical results of Problem 3.

\begin{tabular}{ccccrr}
\hline$[1, u]$ & \multicolumn{2}{c}{ OSN } & & \multicolumn{2}{c}{ NLM } \\
& Initial point & Iter & $\|H(x)\|$ & Iter & $17^{*}$ \\
& $(1,2,3,1)^{T}$ & $17^{*}$ & $8.3645 e-05$ & $2.5678 e-09$ \\
{$\left[0,10^{5}\right]$} & $(3,4,5,6)^{T}$ & $17^{*}$ & $5.5006 e-08$ & $16^{* *}$ & $7.9148 e-07$ \\
& $(10,10,10,10)^{T}$ & - & - & $21^{*}$ & $3.3521 e-08$ \\
& $(100,100,100,100)^{T}$ & $48^{* *}$ & $1.6079 e-04$ & $19^{* *}$ & $1.8672 e-07$ \\
& $(1000,1000,1000,1000)^{T}$ & - & - & $21^{*}$ & $2.6109 e-07$ \\
\hline
\end{tabular}

Mathematically, for each period $k \in 1,2, \ldots, K$, the model RTP can be expressed as an optimization problem as follows:

$$
\begin{gathered}
\max \sum_{i=1}^{N} U\left(x_{i}(k), \omega_{i}(k)\right)-C_{k}\left(L_{k}\right), \\
\text { s.t. } \sum_{i=1}^{N} x_{i}(k) \leq L_{k}, \quad k=1,2, \ldots, K, \\
\quad x_{i}(k) \geq 0, \quad k=1,2, \ldots, K .
\end{gathered}
$$

Considering a more general form of (40) such that

$$
\begin{gathered}
\min f(x), \\
\text { s.t. } g(x) \leq b, \\
x \geq 0 .
\end{gathered}
$$

If $x$ is the optimization solution, it satisfies the Karush-Kuhn-Tucker (KKT) condition such that

$$
\left\{\begin{array}{l}
\nabla f(x)+\lambda \nabla g(x)=0, \\
\lambda(g(x)-b)=0, \\
\lambda \geq 0, g(x)-b \leq 0
\end{array}\right.
$$

where $\lambda$ is the shadow price in economics, namely, the realtime pricing $[22,23]$. Denoting $G(x, \lambda)=\nabla f(x)+\lambda \nabla g(x)$,
$H(x, \lambda)=-g(x)+b$, we obtain the equivalent mixed complementarity problem of (42) as follows:

$$
\left\{\begin{array}{l}
G(x, \lambda)=0, \\
H(x, \lambda) \geq 0, \\
\lambda^{T} H(x, \lambda)=0 .
\end{array}\right.
$$

Considering a smart grid system in a small area with $N=5$, namely, 5 users, we next show numerical simulation results within a 24-hour time pattern as an evaluation of daily operations. The electricity demand of the five businesses $x_{k, 0}$ is chosen randomly from $[1,20]$; the utility function of the user is chosen as follows:

$$
U(x, \omega)= \begin{cases}\omega x-\frac{\alpha x^{2}}{2}, & \text { if } 0 \leq x<\frac{w}{\alpha}, \\ \frac{w^{2}}{2 \alpha}, & \text { if } x \geq \frac{w}{\alpha},\end{cases}
$$

with $\alpha=0.5, w_{i}(k) \in[1,5]$, and $a_{k}=0.5, b_{k}=c_{k}=0$ in the cost function of the energy provider such that

$$
C\left(L_{k}\right)=a L_{k}^{2}+b L_{k}+c_{k} \text {. }
$$

The initial power supply $L_{k, 0}=\sum_{i=1}^{5} x_{i}^{k, 0}$. To illustrate the effectiveness, we compared the price and the social benefit at 


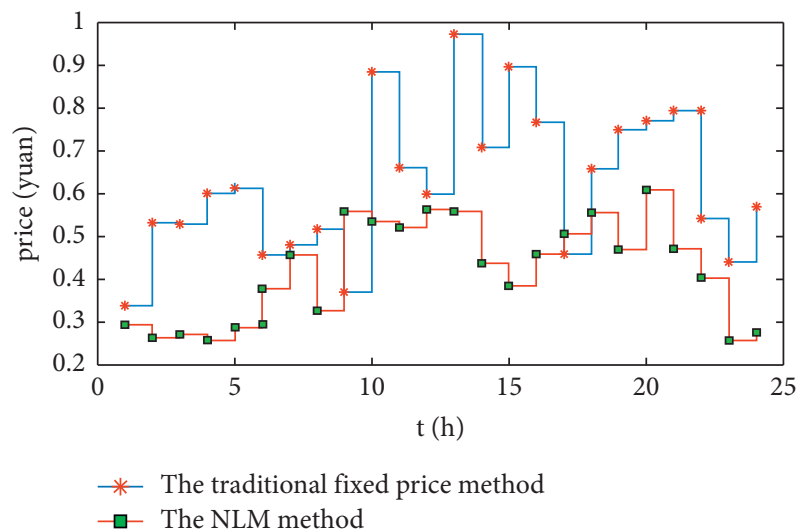

FIgURE 1: The price at different time based on NLM method and traditional fixed price method.

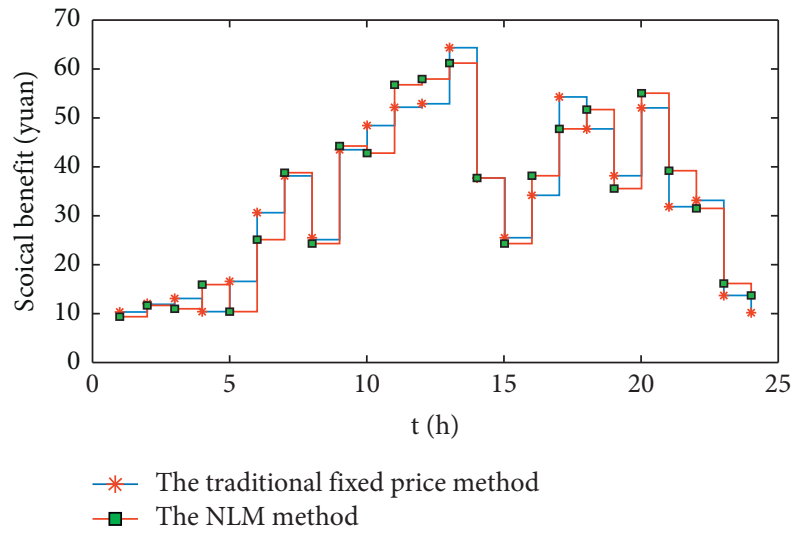

Figure 2: The social benefit at different time based on NLM method and traditional fixed price method.

different time based on the present method (NLM) with those based on the traditional fixed method [16] with $\lambda_{k}=\max \left(w_{k}\right)-\left(a L_{k}\right) / N$. The results are shown in Figures 1 and 2 .

\section{Conclusions}

By using a median function, the mixed complementarity problem is equivalent to a nonsmooth equation. More general, we investigated the generalized Jacobi of a composite vector-valued maximum function, based on which, a modified nonsmooth Levenberg-Marquardt algorithm with a new form LM parameter was proposed. Meanwhile, we discussed the local convergence order of the present algorithm under the local error bound. Compared with the OSN, the present algorithm is more robust while the initial point is far from the solution, it always needs less iterations. While the initial point is near the solution, the present algorithm is also effective, as seen from Tables 1-3. We also discuss the application of the present algorithm for the real-time pricing in this paper. From Figures 1 and 2, we obtain that the electricity price calculated by the present method is significantly lower, and the social benefit is not less than the fixed electricity price method when compared with the traditional fixed price method, which is beneficial to the demand side while not affecting the overall social benefits. Hence, the present algorithm is effective for the real-time price in smart grid. However, the algorithm for the composite vector-valued maximum function is limited, and the subdifferentially regular is a little strong. The calculation of the Clarke Jacobi for other functions and the applications of the present algorithm are our future topics to research.

\section{Data Availability}

This paper includes some numerical examples. It does not include hyperlinks to publicly archived datasets analysed.

\section{Conflicts of Interest}

The authors declare that they have no conflicts of interest.

\section{Acknowledgments}

This work was supported by the National Science Foundation of China (no. 12101198), Henan University Key Research Projects (no. 19A110016), and Henan Scientific and Technological Research Projects (no. 192102210114).

\section{References}

[1] M. C. Ferris and J. S. Pang, "Engineering and economic applications of complementarity problem," SIAM Review, vol. 39, no. 4, pp. 669-713, 1997.

[2] P. T. Harker and J. S. Pang, "Finite-dimensional variational inequality and nonlinear complementarity problems: a survey of theory,algorithms and applications," Mathematical Programming, vol. 48, no. 1-3, pp. 161-220, 1990.

[3] H. Wang and Y. Gao, "Real-time pricing method for smart grids based on complementarity problem," Journal of Modern Power Systems and Clean Energy, vol. 7, no. 5, pp. 1280-1293, 2019.

[4] K. Wang, Z. Zheng, L. Xu, and Y. Li, "Neutral-point voltage balancing method for five-level NPC inverters based on carrier-overlapped PWM," IEEE Transactions on Power Electronics, vol. 36, no. 2, pp. 1428-1440, 2021.

[5] L. Zhang, S. Ji, S. Gu et al., "Design considerations for highvoltage-insulated gate driver power supply for $10-\mathrm{kV} \mathrm{SiC}$ MOSFET applied in medium-voltage converter," IEEE Transactions on Industrial Electronics, vol. 68, no. 7, pp. 5712-5724, 2021.

[6] L. Zhang, X. B. Ruan, and X. Y. Ren, "Second-harmonic current reduction for two-stage single-phase inverter with boost-derived front-end converter: control schemes and design considerations," IEEE Transactions on Power Electronics, vol. 33, no. 7, 2018.

[7] Q. Sun, R. Han, H. Zhang, J. Zhou, and J. M. Guerrero, “A multiagent-based consensus algorithm for distributed coordinated control of distributed generators in the energy internet," IEEE Transactions on Smart Grid, vol. 6, no. 6, pp. 3006-3019, 2015.

[8] P. Samadi, A. H. Mohsenian-Rad, R. Schober, V. W. S. Wong, and J. Jatskevich, "Optimal real-time pricing algorithm based on utility maximization for smart grid," in Proceedings of the IEEE International Conference on Smart Grid Communications, pp. 415-420, Gaithersburg, MD, USA, October 2010. 
[9] P. Samadi, H. Mohsenian-Rad, R. Schober, and V. W. S. Wong, "Advanced demand side management for the future smart grid using mechanism design," IEEE Transactions on Smart Grid, vol. 3, no. 3, pp. 1170-1180, 2012.

[10] A.-H. Mohsenian-Rad, V. W. S. Wong, J. Jatskevich, R. Schober, and A. Leon-Garcia, "Autonomous demand-side management based on gametheoretic energy consumption scheduling for the future smart grid," IEEE Transactions on Smart Grid, vol. 1, no. 3, pp. 320-331, 2010.

[11] W. Zhang, J. Li, G. Chen, Z. Y. Dong, and K. P. Wong, “A comprehensive model with fast solver for optimal energy scheduling in RTP environment," IEEE Transactions on Smart Grid, vol. 8, no. 5, pp. 2314-2323, 2017.

[12] H. B. Zhu, Y. Gao, Y. Hou, and L. Tao, "Real-time pricing considering different type of users based on Markov decision processes in smart grid," Systems Engineering-Theory \& Practice, vol. 38, no. 3, pp. 807-816, 2018.

[13] H. J Wang and Y. Gao, "Research on the real-time pricing of smart grid based on nonsmooth equations," Journal of Systems Engineering, vol. 33, no. 3, pp. 34-41, 2018.

[14] S. Gabriel and J. Moré, Smoothing of Mixed Complementarity Problems in Complementarity and Variational Problems, In State.Art SIAM., Philadelphia, PA, USA, 1997.

[15] Z. Y. Zhou and Y. C. Peng, "The locally Chen-Harker-Kanzow-Smale smoothing functions optimization," Journal of Global Optimization, vol. 74, no. 2, pp. 169-193, 2019.

[16] B. Chen, P. T. Harker, and M. Ç. Pınar, "Continuation methods for nonlinear complementarity problems via normal maps," European Journal of Operational Research, vol. 116, no. 3, pp. 591-606, 1999.

[17] L. Qi, D. Sun, and G. Zhou, “A new look at smoothing Newton methods for nonlinear complementarity problems and box constrained variational inequalities," Mathematical Programming, vol. 87, no. 1, pp. 1-35, 2000.

[18] B. Fan, "A nonmonotone Levenberg-Marquardt method for nonlinear complementarity problems under local error bound," Computational and Applied Mathematics, vol. 36, no. 1, pp. 185-202, 2017.

[19] K. Amini and F. Rostami, "A modified two steps LevenbergMarquardt method for nonlinear equations," Journal of Computational and Applied Mathematics, vol. 288, pp. 341-350, 2015.

[20] L. Song and Y. Gao, "A nonsmooth Levenberg-Marquardt method for vertical complementarity problems," Numerical Algorithms, vol. 76, no. 2, pp. 473-485, 2017.

[21] J. Tang, S. Liu, and C. Ma, "One-step smoothing Newton method for solving the mixed complementarity problem with a P0 function," Applied Mathematics and Computation, vol. 215, no. 6, pp. 2326-2336, 2009.

[22] Y. Gao, "Real-time pricing strategy for smart grid with user of non-decreasing marginal benefit," Journal of Industrial Engineering and Management, vol. 23, no. 1, pp. 10-13, 2018.

[23] Y. M. Dai and Y. Gao, "Real-time pricing decision of smart grid with multiple resources and users," Systems EngineeringTheory \& Practice, vol. 35, no. 9, pp. 2315-2323, 2015. 